\title{
Graph Embedding Using Commute Time
}

\author{
Huaijun Qiu and Edwin R. Hancock \\ Department of Computer Science, University of York, \\ York, YO10 5DD, UK
}

\begin{abstract}
This paper explores the use of commute-time preserving embedding as means of data-clustering. Commute time is a measure of the time taken for a random walk to set-out and return between a pair of nodes on a graph. It may be computed from the spectrum of the Laplacian matrix. Since the commute time is averaged over all potential paths between a pair of nodes, it is potentially robust to variations in graph structure due to edge insertions or deletions. Here we demonstrate how nodes of a graph can be embedded in a vector space in a manner that preserves commute time. We present a number of important properties of the embedding. We experiment with the method for separating object motions in image sequences.
\end{abstract}

\section{Introduction}

The embedding of the nodes of a graph in a vector-space is an important step in developing structural pattern analysis algorithms. For instance, node embeddings are key for graph matching [17,5,4], graph-based clustering [18] and graph visualisation [9]. Although the embedding can be effected using a number of different techniques including those that are geometrically based [19] and those that are based on optimisation techniques [14], one of the simplest approaches is to adopt a graph-spectral approach $[11,1]$. This involves embedding the nodes of the graph under study using the eigenvectors or scaled eigenvectors of the Laplacian or adjacency matrix. For instance both Shapiro and Brady [17], and Kosinov and Caelli [4] use spectral methods to embed nodes of graphs in a vector space, and then use proximity to establish correspondences. Spectral embeddings have also been used to visualise complex graphs.

However, one of the problems of spectral embedding is stability under noise. From matrix perturbation it is well known that noise in an adjacency matrix causes the eigenvectors can rotate erratically under noise, and this means that the embedding coordinates are also unstable under noise. The aim in this paper is to explore the use of commute time as a means of stabilising the spectral embedding of graph nodes. The commute time between a pair of nodes on a graph is the expected time taken for a random walk to set-out and return. It is hence averaged over the set of all possible paths between each pair of nodes. In fact, commute time is a metric that can be computed using the Green's function or pseudo inverse of the graph Laplacian. In a recent series of papers Qiu and Hancock [13] have shown how commute time can give improved graph partitions and spectral clusterings.

The aim in this paper is to investigate whether the averaging of paths that is implicit to the computation of commute time can lead to improved embeddings. The embedding that preserves commute times is found the scaling the transpose of the Laplacian 
eigenvector matrix by the pseudo-inverse of the Laplacian eigenvalues. We commence by performing a theoretical analysis that establishes the link between this embedding and the Laplacian eigenmap and the diffusion map. We then present some experiments that illustrate the practical utility of the embedding.

\section{Commute Time and Commute Time Embedding}

We denote a weighted graph by $\Gamma=(V, E)$ where $V$ is the set of nodes and $E \subseteq V \times V$ is the set of edges. Let $\Omega$ be the weighted adjacency matrix satisfying

$$
\Omega(u, v)= \begin{cases}w(u, v) & \text { if }(u, v) \in E \\ 0 & \text { otherwise }\end{cases}
$$

Further let $T=\operatorname{diag}\left(d_{v} ; v \in V\right)$ be the diagonal weighted degree matrix with elements $d_{u}=\sum_{v=1}^{|V|} w(u, v)$. The un-normalized Laplacian matrix is given by $L=T-\Omega$ and the normalized Laplacian matrix is defined to be $\mathcal{L}=T^{-1 / 2} L T^{-1 / 2}$, and has elements

$$
\mathcal{L}_{\Gamma}(u, v)= \begin{cases}1 & \text { if } u=v \\ -\frac{w(u, v)}{\sqrt{d_{u} d_{v}}} & \text { if } u \neq v \text { and }(u, v) \in E \\ 0 & \text { otherwise }\end{cases}
$$

The spectral decomposition of the normalized Laplacian is $\mathcal{L}=\Phi \Lambda \Phi^{T}$, where $\Lambda=$ $\operatorname{diag}\left(\lambda_{1}, \lambda_{2}, \ldots, \lambda_{|V|}\right)$ is the diagonal matrix with the ordered eigenvalues as elements satisfying: $0=\lambda_{1} \leq \lambda_{2} \ldots \leq \lambda_{|V|}$ and $\Phi=\left(\phi_{1}\left|\phi_{2}\right| \ldots \mid \phi_{|V|}\right)$ is the matrix with the ordered eigenvectors as columns.

Let $G$ be the pseudo-inverse of the normalized Laplacian matrix satisfying $G \mathcal{L}=$ $\mathcal{L} G=I-\phi_{1} \phi_{1}^{T}$. Then we have

$$
G(u, v)=\sum_{i=2}^{|V|} \frac{1}{\lambda_{i}} \phi_{i}(u) \phi_{i}(v)
$$

From Equation 1, the eigenvalues of $\mathcal{L}$ and $G$ have the same sign and $\mathcal{L}$ is positive semidefinite, and so $G$ is also positive semidefinite. Since $G$ is also symmetric (see [6] page 4), it follows that $G$ is a kernel.

We note that the commute time $C T(u, v)$ is the expected time for the random walk to travel from node $u$ to reach node $v$ and then return. It can be computed using the Green's function $G$ by

$$
C T(u, v)=\operatorname{vol} T^{-1 / 2}(G(u, u)+G(v, v)-2 G(u, v)) T^{-1 / 2}
$$

As a result,

$$
C T(u, v)=\sum_{i=2}^{|V|}\left(\sqrt{\frac{v o l}{\lambda_{i} d_{u}}} \phi_{i}(u)-\sqrt{\frac{v o l}{\lambda_{i} d_{v}}} \phi_{i}(v)\right)^{2}
$$

Hence, the embedding of the nodes of the graph into a vector space that preserves commute time has the co-ordinate matrix

$$
\Theta=\sqrt{v o l} \Lambda^{-1 / 2} \Phi^{T} T^{-1 / 2}
$$


The columns of the matrix are vectors of embedding co-ordinates for the nodes of the graph. The term $T^{-1 / 2}$ arises from the normalisation of the Laplacian. The embedding is nonlinear in the eigenvalues of the Laplacian. This distinguishes it from principle components analysis (PCA) and locality preserving projection (LPP) [10] which are both linear. As we will demonstrate in the next section, the commute time embedding is just kernel PCA [16] on the Green's function. Moreover, it can be viewed as Laplacian eigenmap since they actually are minimizing the same objective function.

\subsection{The Commute Time Embedding and the Laplacian Eigenmap}

In the Laplacian eigenmap [3,2] the aim is to embed a set of points with co-ordinate matrix $\overline{\mathbf{X}}=\left\{\overline{\mathbf{x}}_{1}, \overline{\mathbf{x}}_{2}, \ldots, \overline{\mathbf{x}}_{n}\right\}$ from a $R^{n}$ space into a lower dimensional subspace $R^{m}$ with the co-ordinate matrix $\mathbf{Z}=\left\{\mathbf{z}_{1}, \mathbf{z}_{2}, \ldots, \mathbf{z}_{m}\right\}$. The original data-points have a proximity weight matrix $\Omega$ with elements $\Omega(j, j)=\exp \left[-\left\|\overline{\mathbf{x}}_{i}-\overline{\mathbf{x}}_{j}\right\|^{2}\right]$. The aim is to find the embedding that minimises the objective function

$$
\epsilon=\sum_{i, j}\left\|\mathbf{z}_{i}-\mathbf{z}_{j}\right\|^{2} \Omega(i, j)=\operatorname{tr}\left(\mathbf{Z}^{T} L \mathbf{Z}\right)
$$

where $\Omega$ is the edge weight matrix of the original data $\overline{\mathbf{X}}$.

To remove the arbitrary scaling factor and to avoid the embedding undergoing dimensionality collapse, the constraint $\mathbf{Z}^{T} T \mathbf{Z}=I$ is applied. The embedding problem becomes

$$
\mathbf{Z}=\arg \min _{\mathbf{Z}^{T} T \mathbf{Z}=I} \operatorname{tr}\left(\mathbf{Z}^{T} L \mathbf{Z}\right)
$$

The solution is given by the lowest eigenvectors of the generalized eigen-problem

$$
L \mathbf{Z}=\Lambda T \mathbf{Z}
$$

and the value of the objective function corresponding to the solution is $\epsilon^{*}=\operatorname{tr}(\Lambda)$.

For the commute-time embedding the objective function minimised is

$$
\epsilon=\frac{\sum_{i, j}\left\|\mathbf{z}_{i}-\mathbf{z}_{j}\right\|^{2} \Omega_{(i, j)}}{\sum_{i} \mathbf{z}_{i}^{2} d_{i}}=\operatorname{tr}\left(\frac{\mathbf{Z}^{T} L \mathbf{Z}}{\mathbf{Z}^{T} T \mathbf{Z}}\right)
$$

To show this, let $\mathbf{Z}=Y^{T}=\left(\sqrt{\operatorname{vol}} \Lambda^{-1 / 2} \Phi^{T} T^{-1 / 2}\right)^{T}$, we have

$$
\begin{aligned}
\epsilon & =\operatorname{tr}\left(\frac{\sqrt{v o l} \Lambda^{-1 / 2} \Phi^{T} T^{-1 / 2} L T^{-1 / 2} \Phi \Lambda^{-1 / 2} \sqrt{v o l}}{\sqrt{v o l} \Lambda^{-1 / 2} \Phi^{T} T^{-1 / 2} T T^{-1 / 2} \Phi \Lambda^{-1 / 2} \sqrt{v o l}}\right) \\
& =\operatorname{tr}\left(\frac{\Lambda^{-1 / 2} \Phi^{T} \mathcal{L} \Phi \Lambda^{-1 / 2}}{\Lambda^{-1 / 2} \Phi^{T} \Phi \Lambda^{-1 / 2}}\right) \\
& =\operatorname{tr}\left(\frac{\Lambda^{-1 / 2} \Lambda \Lambda^{-1 / 2}}{\Lambda^{-1}}\right) \\
& =\operatorname{tr}(\Lambda)=\epsilon^{*}
\end{aligned}
$$

Hence, the commute time embedding not only aims to maintain proximity relationships by minimizing $\sum_{u, v}\left\|\mathbf{z}_{u}-\mathbf{z}_{v}\right\|^{2} \Omega_{u, v}$, but it also aims to assign large co-ordinate 
values to nodes (or points) with large degree (i.e. it maximizes $\sum_{u} \mathbf{z}_{u}^{2} d_{u}$ ). Nodes with large degree are the most significant in a graph since they have the largest number or total weight of connecting edges. In the commute time embedding, these nodes are furthest away from the origin and are hence unlikely to be close to one-another.

Finally, we note that the objective function appearing in Equation (15) is identical to that used in the normalized cut. To show this let $\boldsymbol{\theta}$ be a dimensional indicator vector. The minimum value obtained by the normalized cut [18] is

$$
\boldsymbol{\theta}_{1}=\arg \min _{\boldsymbol{\theta}^{\boldsymbol{T}_{\mathbf{T}}=0}} \frac{\boldsymbol{\theta}^{\boldsymbol{T}}(\mathbf{T}-\Omega) \boldsymbol{\theta}}{\boldsymbol{\theta}^{\boldsymbol{T}} \mathbf{T} \boldsymbol{\theta}}
$$

Hence comparing with Equation (8) it is clear that the objective function minimised by the commute time embedding is exactly the same as that minimized by the normalized cut, provided that the eigenvectors are scaled by the inverse of the corresponding nonzero eigenvalues. In the bipartition case, this does not make any difference since scaling will not change the distribution of the eigenvector components. However, in the multipartition case, the scaling differentiates the importance of different eigenvectors. It is clear that the eigenvector corresponding to the smallest non-zero eigenvalue contributes the greatest amount to the commute time. Moreover, it is this eigenvector or Fiedler vector that is used in the normalized cut to bipartition the graphs recursively. In the case of the commute time embedding, the scaled eigenvectors are used as projection axes for the data. As a result if we project the data into the commute time embedding subspace, the normalized cut bipartition can be realized by simply dividing the projected data into two along the axis spanned by the Fiedler vector. Further partitions can be realized by projecting and dividing along the axes corresponding to the different scaled eigenvectors.

In Figure 2 we compare the result of embedding using commute time and the Laplacian eigenmap on a planar graph shown in Figure 1. The original graph is constructed by connecting two randomly generated planar graphs. The graph is un-weighted. We project the nodes of the graph onto the plane spanned by the two principle eigenvectors of the mapping. ¿From the figure, it is clear that both embeddings maintain the original graph structure, and that the two original graphs are well separated. However, compared to the Laplacian embedding, the points in the two original graphs are more densely distributed by the commute time embedding. Another significant advantage of the commute time embedding is that it preserves variance in a maximal way. This is illustrated in 2(b). Here two randomly generated graphs are embedded in two orthogonal planes.

\subsection{The Commute Time and the Diffusion Map}

Finally, it is interesting to note the relationship with the diffusion map embedding of Lafon et al [15]. The method commences from the random walk on a graph which has transition probability matrix $P=T^{-1} A$, where $A$ is the adjacency matrix. Although $P$ is not symmetric, it does have a right eigenvector matrix $\Psi$, which satisfies the equation

$$
P \Psi=\Lambda_{P} \Psi
$$




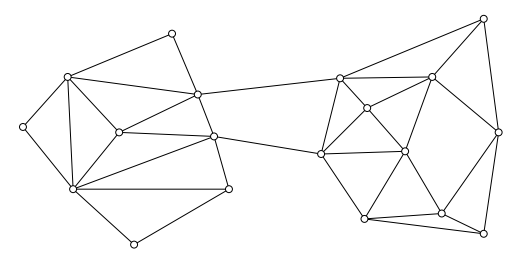

Fig. 1. Original planar graph

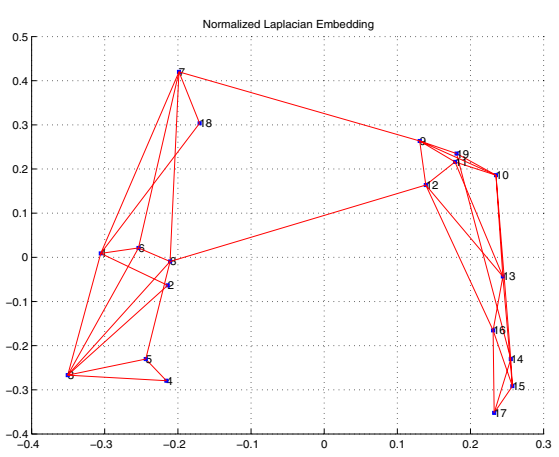

(a) Normalised Laplacian Embedding.

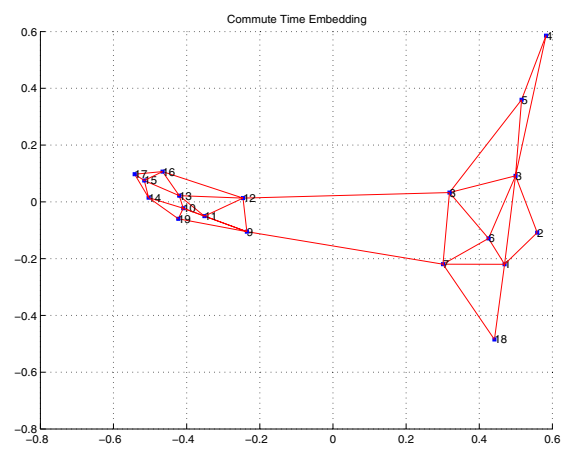

(b) Commute Time Embedding.

Fig. 2. Graph embedding comparison

Since $P=T^{-1} A=T^{-1}(T-L)=I-T^{-1} L$ and as result

$$
\begin{aligned}
\left(I-T^{-1} L\right) \Psi & =\Lambda_{P} \Psi \\
T^{-1} L \Psi & =\left(I-\Lambda_{P}\right) \Psi \\
L \Psi & =\left(I-\Lambda_{P}\right) T \Psi
\end{aligned}
$$

which is identical to Equation 7 if $\mathbf{Z}=\Psi$ and $\Lambda=I-\Lambda_{P}$ The embedding co-ordinate matrix for the diffusion map is $Y=\Lambda^{t} \Psi^{T}$, where $t$ is real. For the embedding the diffusion distance between a pair of nodes is

$$
D_{t}^{2}(u, v)=\sum_{i=1}^{m}\left(\lambda_{P}\right)_{i}^{2 t}\left(\psi_{i}(u)-\psi_{i}(v)\right)^{2}
$$

Clearly if we take $t=-1 / 2$ the diffusion map is equivalent to the commute time embedding and the diffusion time is equal to the commute time.

The diffusion map is designed to give a distance function that reflects the connectivity of the original graph or point-set. The distance should be small if a pair of points are connected by many short paths, and this is also the behaviour of the commute time. The advantage of the diffusion map or distance is that it has a free parameter $t$, and this may be varied to alter the properties of the map. The disadvantage is that when $t$ is small, the diffusion distance is ill-posed. The reason for this is that according to the original definition of the diffusion distance for a random walk $\left(D_{t}^{2}(u, v)=\left\|p_{t}(u, \cdot)-p_{t}(v, \cdot)\right\|^{2}\right)$, 
and as a result the distance between a pair of nodes depends on the transition probability between the nodes under consideration and all of the remaining nodes in the graph. As a result if $t$ is small, then the random walk will not have propagated significantly, and the distance will depend only on very local information. There are also problems when $t$ is large. When this is the case the random walk converges to its stationary state with $P^{t}=T /$ vol ( a diagonal matrix), and this gives zero diffusion distance for all pairs of distinct nodes. So it is a critical to control $t$ carefully in order to obtain useful embeddings.

\section{Multi-body Motion Tracking Using Commute Time}

In this section, we will show how the multi-body motion tracking problem can be posed as one of commute time embedding. Suppose there are $N$ objects moving independently in a scene and the movement is acquired by an affine camera as $F$ frames. In each frame, $P$ feature points are tracked and the coordinate of the $i$ th point in the $f$ th frame is given by $\left(x_{i}^{f}, y_{i}^{f}\right)$. Let $X$ and $Y$ denote two $F \times P$ matrices constructed from the image coordinates of all the points across all of the frames:

$$
X=\left[\begin{array}{cccc}
x_{1}^{1} & x_{2}^{1} & \cdots & x_{P}^{1} \\
x_{1}^{2} & x_{2}^{2} & \cdots & x_{P}^{2} \\
\vdots & \vdots & \ddots & \vdots \\
x_{1}^{F} & x_{2}^{F} & \cdots & x_{P}^{F}
\end{array}\right] \quad Y=\left[\begin{array}{cccc}
y_{1}^{1} & y_{2}^{1} & \cdots & y_{P}^{1} \\
y_{1}^{2} & y_{2}^{2} & \cdots & y_{P}^{2} \\
\vdots & \vdots & \ddots & \vdots \\
y_{1}^{F} & y_{2}^{F} & \cdots & y_{P}^{F}
\end{array}\right]
$$

Each row in the two matrices above corresponds to a single frame and each column corresponds to a single point. The two coordinate matrices can be stacked to form the matrix

$$
W=\left[\frac{X}{Y}\right]_{2 F \times P}
$$

The $W$ matrix can be factorized into a motion matrix $M$ and a shape matrix $S$ thus, $W_{2 F \times P}=M_{2 F \times r} \times S_{r \times P}$ where $r$ is the rank of $W(r=4$ in the case of $W$ without noise and outliers). In order to solve the factorization problem, matrix $W$ can be decomposed by SVD:

$$
W=U \Sigma R^{T}
$$

If the features from the same object are grouped together, then $U, \Sigma$ and $R$ will have a block-diagonal structure.

$$
W=\left[U_{1} \cdots U_{N}\right]\left[\begin{array}{ccc}
\Sigma_{1} & & \\
& \ddots & \\
& & \Sigma_{N}
\end{array}\right]\left[\begin{array}{lll}
R_{1}^{T} & & \\
& \ddots & \\
& & R_{N}^{T}
\end{array}\right]
$$

and the shape matrix for object $k$ can be approximated by $S_{k}=B^{-1} \Sigma_{k} R_{k}^{T}$ where $B$ is an invertible matrix that can be found from $M$.

In a real multi-body tracking problem, the coordinates of the different objects are potentially permuted into a random order. As a result it is impossible to correctly recover the shape matrix $S_{k}$ without knowledge of the correspondence order. Since the 
eigenvector matrix $V$ is related to the shape matrix, the shape interaction matrix was introduced by Costeira and Kanade [8,7] to solve the multi-body separation problem. The shape interaction matrix is

$$
Q=R R^{T}=\left[\begin{array}{cccc}
S_{1}^{T} \Sigma_{1}^{-1} S_{1} & 0 & \cdots & 0 \\
0 & S_{2}^{T} \Sigma_{2}^{-1} S_{2} & \cdots & 0 \\
\vdots & \vdots & \ddots & 0 \\
0 & 0 & \cdots & S_{N}^{T} \Sigma_{N}^{-1} S_{N}
\end{array}\right]
$$

¿From Equation 13, the shape interaction matrix $Q$ has the convenient properties that $Q(u, v)=0$, if points $u, v$ belong to different objects and $Q(u, v) \neq 0$, if points $u, v$ belong to the same object. The matrix $Q$ is also invariant to both the object motion and the selection of the object coordinate systems.

Our aim is to use commute time as a shape separation measure. Specifically, we use the commute time to refine the block structure of the $Q$ matrix and group the feature points into objects.

\section{Object Separation Steps}

The algorithm we propose for this purpose has the following steps:

1. Use the shape interaction matrix $Q$ as the weighted adjacency matrix $\Omega$ and construct the corresponding graph $\Gamma$.

2. Compute the Laplacian matrix of graph $\Gamma$ using $L=T-Q$.

3. Find the eigenvalue matrix $\Lambda$ and eigenvector matrix $\Phi$ of $L$ using $L=\Phi \Lambda \Phi^{T}$.

4. Compute the commute time matrix $C T$ using $\Lambda$ and $\Phi$ from Equation (3).

5. Embed the commute time into a subspace of $R^{n}$ using Equation (4).

6. Cluster the data points in the subspace using the k-means algorithm [12].

\section{Experiments}

We commence in Figure 3 by showing four synthetic examples of point-configurations (left-hand panel) and the resulting commute time embeddings (right-hand panel). Here we have computed the proximity weight matrix $\Omega$ by exponentiating the Euclidean distance between points. The main features to note are as follows. First, the embedded points corresponding to the same point-clusters are cohesive, being scattered around approximately straight lines in the subspace. Second, the clusters corresponding to different objects give rise to straight lines that are orthogonal.

Turning our attention to the multi-body tracking example, the top row of Figure 4 shows images from five real-world video sequences. In the second row of the figure, we show the trajectories for the tracked points in each of the video sequences. Here the outliers are successfully removed. The different sequences offer tasks of increasing difficulty. The easiest sequence is the one labelled $\mathbf{A}$, where background has a uniform and almost linear relative movement, and the foreground car follows a curved trajectory. There is a similar pattern in the sequence labelled $\mathbf{B}$, but here the background movement is more significant. In sequence $\mathbf{C}$, there is both camera pan and abrupt object 

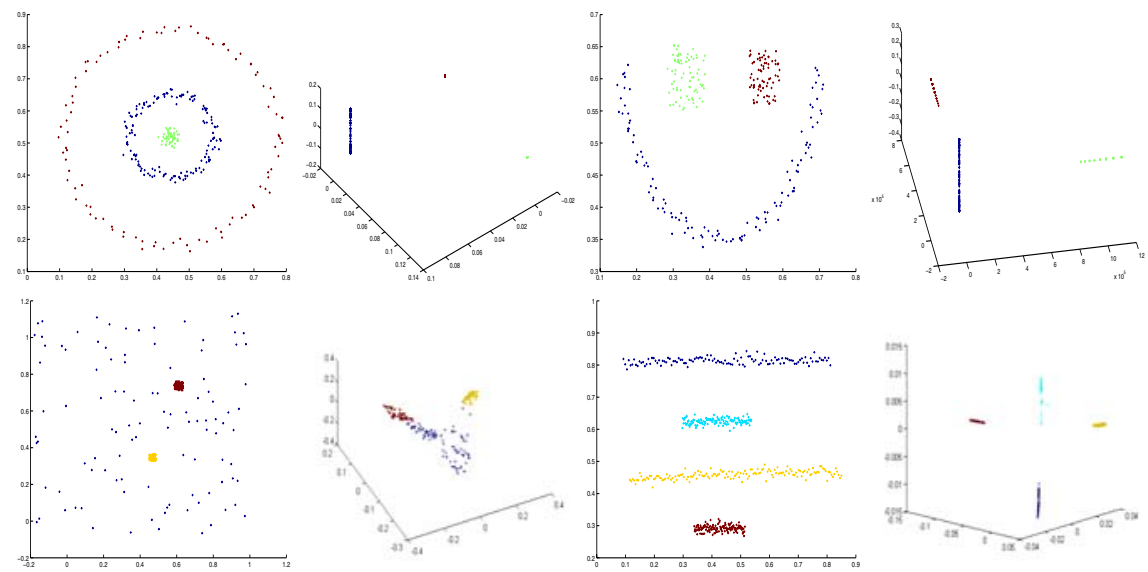

Fig. 3. Commute time embedding examples

movement. Sequence $\mathbf{D}$ has camera pan and three independently moving objects. In sequence $\mathbf{E}$ there is background jitter (due to camera shake) and two objects exhibiting independent overall movements together with articulations. Finally, in the third row of the figure, we show the embeddings of the tracked points for the sequences. The feature to note, is that the different moving objects form distinct clusters and are well separated from the background. The colour coding scheme used in the plot is the same as that used in the fifth column of Figure 4.
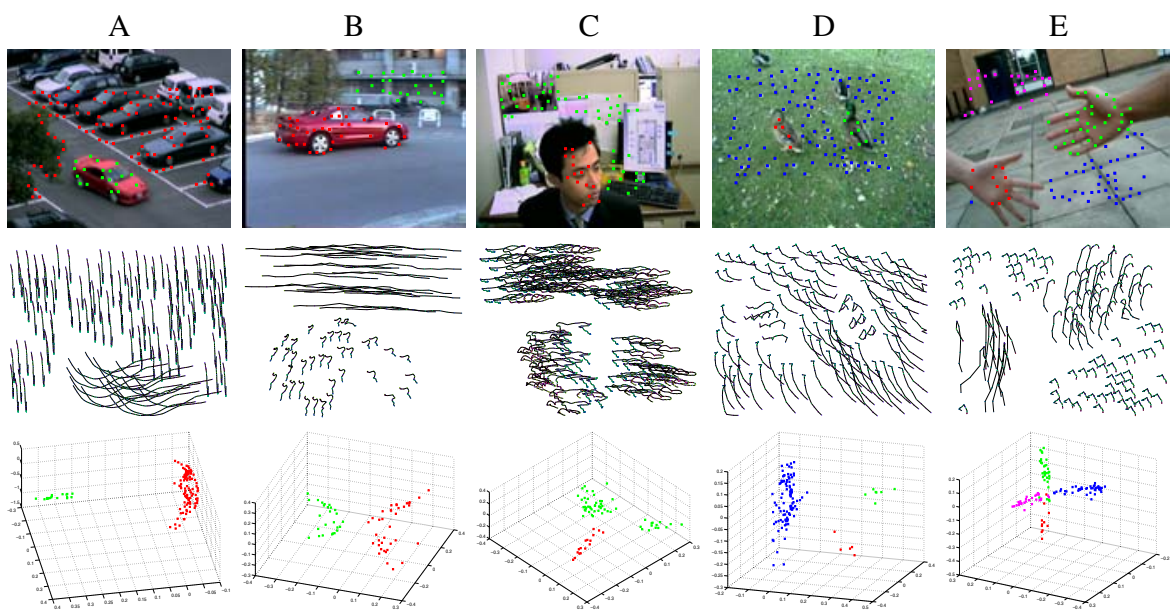

Fig. 4. Real-world video sequences and successfully tracked feature points 


\section{Conclusion}

We have explored the theoretical properties of the commute time embedding, and have established a link with a number of alternative methods in the manifold learning literature. Experiment results show that the embedding maps different point clusters into approximately linear subspaces, that can be easily separated.

\section{References}

1. X. Bai, H. Yu, and E.R. Hancock. Graph matching using spectral embedding and alignment. In $I C P R$, pages 398-401, 2004.

2. M. Belkin and P. Niyogi. Laplacian eigenmaps and spectral techniques for embedding and clustering. In Advances in Neural Information Processing Systems, pages 585-591, 2001.

3. M. Belkin and P. Niyogi. Laplacian eigenmaps for dimensionality reduction and data representation. Neural Computation, 15(6):1373-1396, 2003.

4. T. Caelli and S. Kosinov. An eigenspace projection clustering method for inexact graph matching. IEEE Trans. Pattern Anal. Mach. Intell., 26(4):515-519, 2004.

5. M. Carcassoni and E.R. Hancock. Spectral correspondence for point pattern matching. Pattern Recognition, 36(1):193-204, 2003.

6. F.R.K. Chung and S.-T. Yau. Discrete green's functions. In J. Combin. Theory Ser, pages 191-214, 2000.

7. J. Costeira and T. Kanade. A multi-body factorization method for motion analysis. In ICCV, pages 1071-1076, 1995.

8. J. Costeira and T. Kanade. A multibody factorization method for independently moving objects. IJCV, 29(3):159 - 179, 1997.

9. D. Harel and Y. Koren. Graph drawing by high-dimensional embedding. In GD '02: Revised Papers from the 10th International Symposium on Graph Drawing, pages 207-219, 2002.

10. X. He and P. Niyogi. Locality preserving projections. In NIPS, pages 585-591, 2003.

11. B. Luo, R.C. Wilson, and E.R. Hancock. Spectral embedding of graphs. 2002 Winter Workshop on Computer Vision, 2002.

12. J. B. MacQueen. Some methods for classification and analysis of multivariate observations. In Proceedings of the fifth Berkeley symposium on mathematical statistics and probability, pages 281-297, 1967.

13. H. Qiu and E.R. Hancock. Image segmentation using commute times. In $B M V C$, pages 929-938, 2005.

14. S. Roweis and L. Saul. Nonlinear dimensionality reduction by locally linear embedding. Science, 290(5500):2323-2326, 2000.

15. R.R.Coifman, S. Lafon, A.B. Lee, M. Maggioni, B. Nadler, F. Warner, and S.W. Zucker. Geometric diffusions as a tool for harmonic analysis and structure definition of data: Diffusion maps. National Academy of Sciences, 102(21):7426-7431, 2005.

16. B. Sch, A. Smola, and K. Muller. Nonlinear component analysis as a kernel eigenvalue problem. Neural Computation, 10:1299-1319, 1998.

17. L. Shapiro and J. Brady. Feature-based correspondence: an eigenvector approach. Image and Vision Computing, 10(2):283-288, June 1992.

18. J. Shi and J. Malik. Normalized cuts and image segmentation. IEEE PAMI, 22(8):888-905, 2000.

19. J. B. Tenenbaum, V. de Silva, and J. C. Langford. A global geometric framework for nonlinear dimensionality reduction. Science, 290(5500):2319-2323, 2000. 